\title{
Erbium Laser in Mucocele Surgery
}

\author{
1Jan Vokurka, ${ }^{2}$ Lydie I Holla, ${ }^{3}$ Antonin Fassmann
}

\begin{abstract}
Mucoceles are benign tumors originating from minor salivary glands of the oral cavity. They are localized most frequently on the lower lip or buccal mucosa. Unintentional lip biting, localized trauma, and orthodontic devices are among the most frequent causes of mucoceles. In this case report, successful treatment of medium-sized mucocele with erbium laser is presented. Benefits as well as potential risks of mucocele surgery are discussed.
\end{abstract}

Keywords: Chromium: Yttrium-scandium-gallium-garnet, Erbium, Erbium laser, Mucocele, Oral mucosa lesion, Soft tissue tumor.

How to cite this article: Vokurka J, Holla LI, Fassmann A. Erbium Laser in Mucocele Surgery. Int J Laser Dent 2015; 5(2):50-52.

Source of support: Nil

Conflict of interest: None

\section{INTRODUCTION}

Mucoceles are soft, sessile, mucus-filled cavities that originate from minor salivary glands of the oral cavity. ${ }^{1}$ These benign tumors are round or oval, rise above the surrounding mucosa, and are fluctuant to palpation. Although they are usually painless, mucoceles might be unpleasant and unesthetic for the patient. The predominant localization is on the inferior labial and buccal mucosa. ${ }^{2}$ However, they can also be found in any minor salivary gland on the tongue, soft palate, or the retromolar region. ${ }^{3}$

According to the etiopathogenesis, the lesions can be divided into less frequent retention mucoceles found predominantly in elderly individuals and extravasation mucoceles that affect patients under 30 years of age. Together with traumatic fibromas, they represent the most common soft tissue tumors, ${ }^{4}$ with incidence of 2.5 lesions per 1,000 people.

Mucoceles are filled with viscous fluid mucin and are fluctuant to palpation. Diagnosis is made from the medical history, clinical picture, and histopathology.

\footnotetext{
${ }^{1}$ Assistant Professor, ${ }^{2,3}$ Professor

${ }^{1-3}$ Department of Periodontology, Clinic of Dentistry, Brno, Czech Republic
}

Corresponding Author: Jan Vokurka, Assistant Professor Department of Periodontology, Clinic of Dentistry, Brno, Czech Republic, Phone: +00420543183458, e-mail:vokurec@gmail.com
In the differential diagnosis, we have to consider fibroma, hematoma, hemangioma, lymphangioma, abscess, mucous pemphigoid, and bullous oral lichen planus. In the histopathological picture of extravasation mucoceles, fibrous tissue with no epithelial lining forms a pseudocyst that is filled with mucin. They are predominantly found on the lower labial mucosa. On the contrary, retention mucoceles are true cysts with epithelial lining of squamous or cuboid cells and are evenly distributed among the small salivary glands in the oral cavity. ${ }^{5}$

Mucoceles can rupture spontaneously or after trauma from biting, orthodontic appliance, or intentional selftraumatization. If the fluid content is released, they can diminish in size. Seldom, mucoceles might disappear spontaneously, but in the case of repeated irritation, they relapse often and the lesion may become firmer and more difficult to rupture. ${ }^{6}$

Therapy begins with elimination of the traumatic irritation. Sharp teeth edges are rounded, orthodontic braces are covered, and occlusion adjustments are made to diminish the possible traumatization of the oral mucosa. Various oral guards can be prescribed to separate the oral mucosa from unintentional night biting or orthodontic devices. Removal of the mucocele includes marsupialization and extirpation in toto. To diminish the chance for relapse, it is important to remove the whole mucocele together with the salivary gland. Although conventional surgery is used for mucocele removal, various lasers are also suitable for this procedure with several advantages.

Erbium lasers are suitable for procedures on both hard and soft tissues. Our case report was done with erbium, chromium: Yttrium-scandium-gallium-garnet (Er,Cr:YSGG; Biolase Technology; CA, USA) that emits a wavelength of 2,780 $\mathrm{nm}$. The laser emission is enhanced by erbium and chromium in photon-enhanced medium containing yttrium, scandium, gallium, and garnet. ${ }^{7}$

\section{CASE REPORT}

A 24-year-old male patient with noncontributory medical history was referred to the Department of Periodontology. He complained about a soft painless lesion on the lower lip that was slowly growing for the last 4 months (Figs 1 and 2). Patient remarked occasional biting of the lip. After medical history and clinical examination, sharp edges of the front teeth were rounded with soft rubber cup. Silicone night guard was fabricated to protect the 


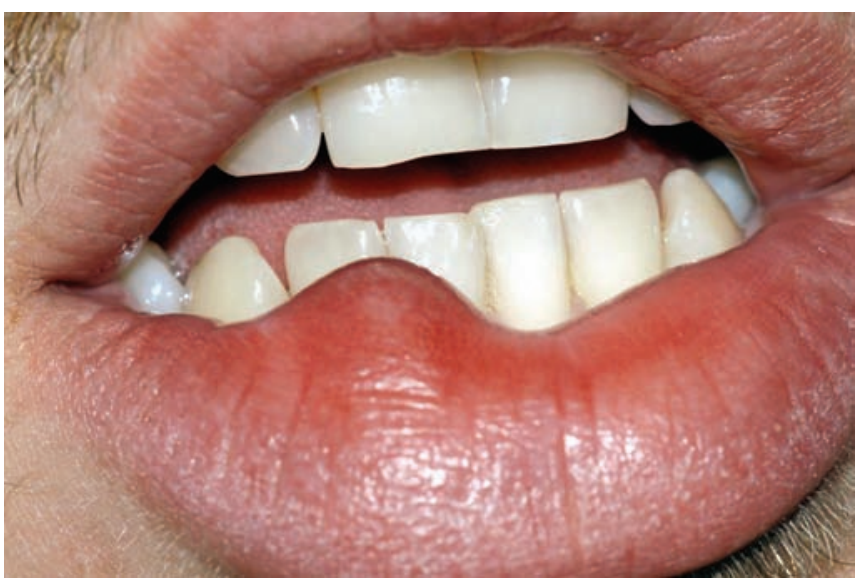

Fig. 1: Lower lip mucocele before the treatment

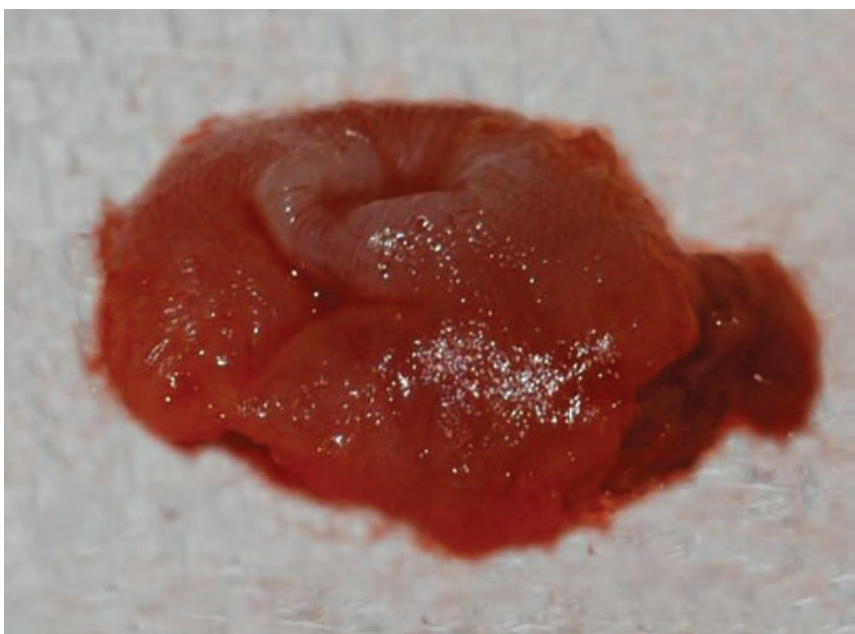

Fig. 3: Biopsy specimen after removal with erbium laser

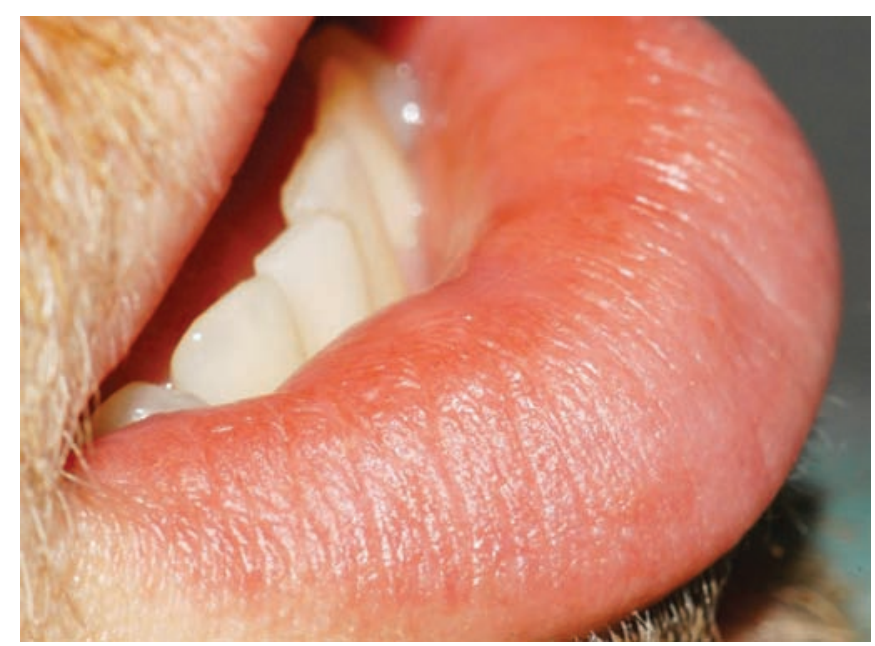

Fig. 5: The healing was uneventful. Natural lip line was reestablished

oral mucosa from unintentional night biting. After consultation with the patient, we recommended extirpation of the tumor with Er,Cr:YSGG laser. The procedure was done infiltrating perilesional local anesthesia using $4 \%$ articaine with 1:100,000 adrenaline. First, the lesion was

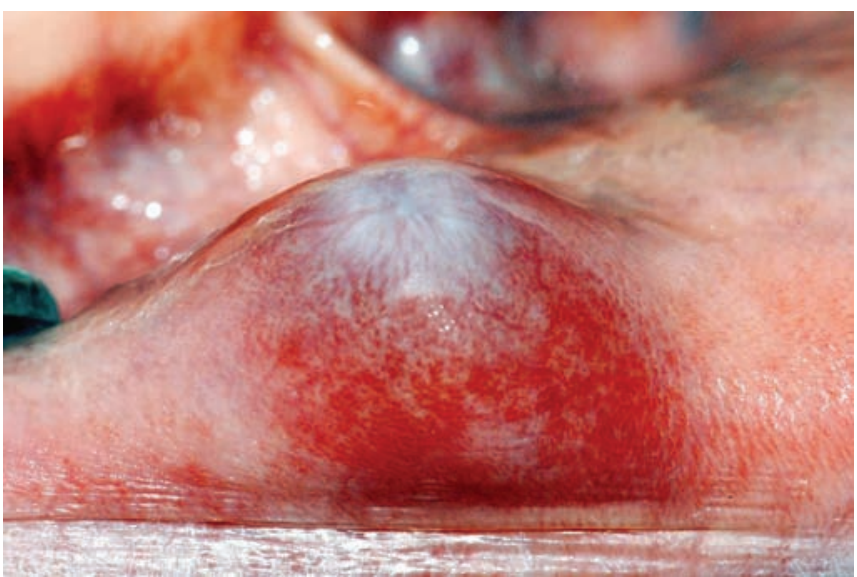

Fig. 2: Details of the mucocele

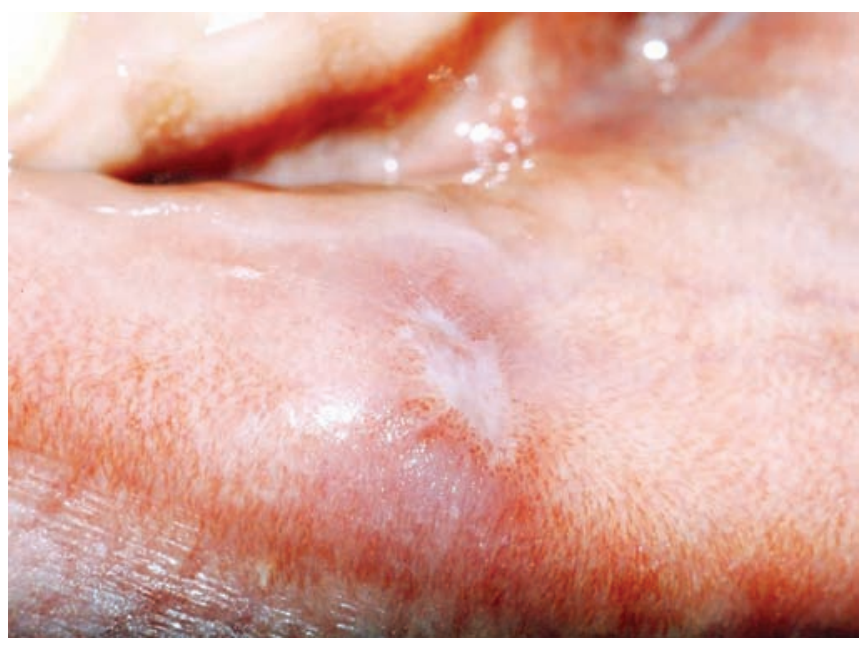

Fig. 4: Two weeks after the surgery, minimal scar was observed

demarcated using $0.5 \mathrm{~W}$ with no water and no air. The mucocele was then carefully removed with focalized beam $2.0 \mathrm{~W}$ with $5 \%$ water and $9 \%$ air. The tumor was removed in toto without rupturing the membrane (Fig. 3). The underlying surface was vaporized with defocalized beam using $0.75 \mathrm{~W}$ with $8 \%$ water and $8 \%$ air. At the end, the surgical wound was disinfected with $0.5 \mathrm{~W}$ with no water and no air for 20 seconds. No anti-inflammatory drugs or antibiotics were prescribed. We recommended $1 \%$ chlorhexidine gel to be applied twice daily and reinforced appropriate oral hygiene with soft toothbrush and interdental tools. The histological observation confirmed the diagnosis of extravasation mucocele. The healing was uneventful and although it was by second intention, minimal scar was observed 2 weeks after the surgery (Figs 4 and 5). There was no relapse of the mucocele.

\section{DISCUSSION}

Mucoceles are common soft tissue tumors that evolve from minor salivary glands. They represent one of the most frequent oral benign tumors of the oral cavity and 
are evenly distributed between males and females. The highest prevalence is reported in the second and third decade of life. Traumatic etiology is confirmed by many authors in the literature. ${ }^{2,8,9}$ Labial mucosa of the lower lip is the most susceptible place for these lesions because of accidental or intentional traumatism. The growth of mucocele is usually slow. The tumor develops during a period of several weeks to several years, with an average of 4 months. ${ }^{10}$

Besides conventional surgery, various techniques using $\mathrm{CO}_{2}$, diode or erbium laser, cryosurgery, micromarsupialization, and intralesional corticoid injection are described in the literature. Conventional surgery requires complete removal of the tumor together with the involved salivary gland. The challenge for the surgeon is the flap closure, which might create unesthetic scars and wound shrinkage. This is most evident in the vermilion area of the lip. Careful suturing of adjacent or transposition flaps may reduce such complications. Removal of the mucocele with laser does not require suturing, leaves no or minimal scaring on the mucosa, allows precise ablation due to its hemostatic properties, and reduces postoperative discomfort and pain. The erbium lasers like Er,Cr:YSGG have minimal thermal damage to the surrounding tissues but have limited hemostatic effect compared with $\mathrm{CO}_{2}$ and diode lasers. ${ }^{11}$ Minimal damage of the biopsy specimen is an advantage of erbium lasers. According to the available literature, all mentioned lasers have minimal postoperative complications and relapses. ${ }^{12}$

In this case report, the successful treatment of a medium-sized mucocele of a young male patient using Er,Cr:YSGG laser was presented. Together with conventional surgery, erbium lasers represent a suitable alternative for mucocele removal. For definitive diagnosis, the importance of histological specimen examination must be stressed.

\section{REFERENCES}

1. Baurmash HD. Mucoceles and ranulas. J Oral Maxillofac Surg 2003 Mar;61(3):369-378.

2. Bahadure RN, Fulzele P, Thosar N, Badole G, Baliga S. Conventional surgical treatment of oral mucocele: a series of 23 cases. Eur J Paediatr Dent 2012 Jun;13(2):143-146.

3. Inoue A, Ikeda S, Mizuno Y, Ogawa H. Superficial mucoceles of the soft palate. Dermatology 2005;210(4):360-362.

4. Baurmash $\mathrm{H}$. The etiology of superficial oral mucoceles. J Oral Maxillofac Surg 2002 Feb;60(2):237-238.

5. Okumura K, Inui M, Nakase M, Nakamura S, Hiramoto K, Tagawa T. A case of submandibular gland mucocele. J Clin Pediatr Dent 2007 Spring;31(3):207-209.

6. Jinbu Y, Kusama M, Itoh H, Matsumoto K, Wang J, Noguchi T. Mucocele of the glands of Blandin-Nuhn: clinical and histopathologic analysis of 26 cases. Oral Surg Oral Med Oral Pathol Oral Radiol Endod 2003 Apr;95(4):467-470.

7. Rizoiu IM, Eversole LR, Kimmel AI. Effects of an erbium, chromium: yttrium, scandium, gallium, garnet laser on mucocutanous soft tissues. Oral Surg Oral Med Oral Pathol Oral Radiol Endod 1996 Oct;82(4):386-395.

8. Boj JR, Poirier C, Espasa E, Hernandez M, Espanya A. Lower lip mucocele treated with an erbium laser. Pediatr Dent 2009 May-Jun;31(3):249-252.

9. Granholm C, Olsson Bergland K, Walhjalt H, Magnusson B. Oral mucoceles; extravasation cysts and retention cysts. A study of 298 cases. Swed Dent J 2009;33(3):125-130.

10. Yagüe-García J, España-Tost AJ, Berini-Aytés L, Gay-Escoda C. Treatment of oral mucocele-scalpel versus $\mathrm{CO}_{2}$ laser. Med Oral Patol Oral Cir Bucal 2009 Sep 1;14(9):e469-e474.

11. Tamarit-Borrás M, Delgado-Molina E, Berini-Aytés L, Gay-Escoda C. Removal of hyperplastic lesions of the oral cavity. A retrospective study of 128 cases. Med Oral Patol Oral Cir Bucal 2005 Mar-Apr;10(2):151-162.

12. Huang IY, Chen CM, Kao YH, Worthington P. Treatment of mucocele of the lower lip with carbon dioxide laser. J Oral Maxillofac Surg 2007 May;65(5):855-858. 